\title{
Verzeich nifs
}

\section{der $\mathrm{Her}$ e $\mathrm{r}$ it a rbeiter.}

Herr Dr. d'Alton, Professor zu Halle. d'A - n.

.. - v. Ammon, Hofrath, Leibarzt und Professor zn Dresden. v. A-n.

- Andresse, pract. Arzt zu Berlin. A-e.

- Balling, Professor zu Würzburg. B - g.

-.. - Barez, Geheimer-Medicinalrath und Professor zu Berlin. B-z.

- - Baumgärtner, Hofrath uud Professor zu Freiburg. B - r.

- Beger, pract. Arzt zu Dresden. Be-r.

- - Berndt, Geheimer-\|ledicinalrath und Professor zu Greifswald. B - $\mathrm{dt}$.

- - Berthold, Professor zu Göttingen. B - d.

- - Burtz, pract. Arzt zu Berlin. B - tz.

- - Bischoff, Professor zu Heidelberg. B - ff.

- - Brandt, Director des zoologischen Museums zn St. Petersburg. Br - dt.

- - von dem Busch, pract. Arzt zu Bremen. v. d. B - sch.

- - Casper, Gebeimer-Medicinalrath und Professor zu Berlin. C-r.

- - Ebermaier, Kreisphysicus zu Düsseldorf. E - r.

- - Eulenburg, pract. Arzt zu Berlin. E-rg.

- - Feist, pract. Arzt zu Mainz. F - st.

- Fest, Regimentsarzt zu Luxemburg. F - t.

- - Fischer, Medicinalrath in Lüneburg. F - r.

- Fraenzel, Leibchirurg u. Regimentsarzt zu Dresden. F - 1 .

- - Froriep, Medicinalrath und Professor zu Berlin. F - p.

- - Geisler, Regimentsarzt zu Lüben. Ge - r.

- - Göschen, pract. Arzt zu Magdeburg. G - n.

- - E. Graefe, Mtdicinalrath und Privatdocent zu Berlin. E. Gr-e.

- - Grojsheim, Medicinalrath u. Regimentsarzt zu Berlin. G - m.

- - Günther, Medicinalrath zu Cöln. Gü - r.

- - Gurlt, Prof. zu Berlin. G - t.

- - Hedenus, pract. Arzt zu Dresden. H - s.

- - Henle, Professor zu Zürich. H - e.

- - Hertwig, Professor zu Berlin. He -- g.

- - Heyfelder, Professor in Erlangen. H - der.

- - Hohl, Professor zu Halle. H - 1 .

- Hollstein, pract. Arzt zu Berlin. H - n.

- - W. Horn, Regierungs-Rath in Erfurt. W. H - n.

- Hüter, Professor zu Marburg. Hü - r.

- Jacobi, Obermedicinalrath und Director der Irrenanstalt zu Siegburg. $\mathbf{J}-\mathbf{i}$.

- Jessen, Director der Irrenanstalt zu Schleswig. J - $\mathbf{n}$.

- Kärnbaçh, pract. Arzt zu Berlin. K-ch. 
Herr Dr. Klose, Professor zu Breslau. Kl - e.

- - v. Köhring, Leibarzt zu Stollberg. v. K - \$.

- - Krombholz, Professor zu Prag. Kr - lz.

- Langenmeyer, Stabsarzt zu Berlin. L - r.

- - Lehfeldt, pract. Arzt zu Berlin. L - dt.

- - Maier, weiland pracl. Arzt zu Berlin. Ma-r.

- - Magnus, prakt. Arzt zu Berlin. MI - s.

- - R. Marchand, Privatdocent zu Berlin. R. M - d.

- - G. Meyer, Stabsarzt zu Berlin. G. M - r.

- - Michaelis, pract. Arzt zu Berlin. II - lis.

- Moser, pract. Arzt zu Berlin. II - r.

- Nagel, pract. Arzt in Berlin. N - I.

- - Naumann, Professor zu Bonn. $\mathrm{Na}-\mathrm{n}$.

- - Neumann, Regierungsrath zu Aachen. Ne - n.

- - Phoebus, pract. Arzt zu Stollberg. $\mathrm{Ph}-\mathrm{s}$.

- Pockels, weiland Generalstabsarzt zu Braunschweig. P - s.

- - Purkinje, Professor zu Breslau. P - e.

- - Rahts, Regiments-Arzt. R - s.

- - Ratzeburg, Professor zu Neustadt.Eberswalde. R -- g.

- - Remack, pract. Arzt zu Berlin. Re-k.

- - v. Schlechtendal, Professor zu Halle. v. Sch - I.

- - Schlemm, Professor zu Berlin. S - m.

- Schotte, Stabsarzt za Berlin. Sch - te.

- - Schöller, pract. Arzt zu Berlin. Sch - r.

- - Schultz, Professor zu Berlin. C. S. - tz.

- - Schwann, Professor zu Löwen. Sch - n.

- - Seifert, Professor zu Greifswald. S - rt.

- - Seiler, Hofrath und Direstor zu Dresden. S - r.

- Siebenhaar, Amtsarzt zu Dresden. Si - r.

- — Ed. v Siebold, Professor zu Göttingen. Ed. v. S - d.

- - Simon, jun., pract. Arzt in Hamburg. S - n. jun.

- Simon, pract. Arzt in Berlin. G. $\mathbf{S}-\mathbf{n}$.

- Simonson, pract. Arzt zu Berlin. S - n.

- - Stannius, Professor zu Rostock. St - s.

- Stark, Geheimer Hofrath und Professor zu Jena. St - rk.

- - Staub, Physicus zu Bamberg. S - b.

- - Steinthal, pract. Arzt zu Berlin. St-l.

- - v. Stosch, Geheimer Rath und Leibarzt za Berlin. v. St - sch.

- - Tott, pract. Arzt zu Rybnick. T - tt.

- - Troschel, Privatdocent za Berlin. T 2 I.

- - Ullmann, Professor zu Marburg. Ull - n.

- - Ulsamer, Professor zu Landshut. U - r.

- - Valentin, Professor za Bern. V - n.

- - Vetter, pract. Arzt zu Berlin. V - r

- Wagner, Geheimer Medicinalrath u. Professor zu Berlin. $\mathrm{Wg} \rightarrow 1$

- - Warnatz, pract. Arzt zu Dresảen. W-tz.

Die Chiffern: B - h., D - ch., H - r., H - m., J - a L - k., J. M - r. und $\mathbf{O}-\mathbf{n}$ zeigen die Namen der Heraugeber 